\title{
Variations
}

Variations

Revue internationale de théorie critique

18 | 2013

"La poésie est une arme chargée de futur »

\section{Politisation de la parole : du rap ludique au rap engagé}

\section{Mathieu Marquet}

\section{(2) OpenEdition}

\section{Journals}

Édition électronique

URL : http://journals.openedition.org/variations/645

DOI : 10.4000/variations. 645

ISSN : 1968-3960

Éditeur

Les amis de Variations

Référence électronique

Mathieu Marquet, « Politisation de la parole : du rap ludique au rap engagé », Variations [En ligne],

18 | 2013, mis en ligne le 31 mai 2013, consulté le 23 avril 2019. URL : http://journals.openedition.org/ variations/645; DOl : 10.4000/variations.645

Ce document a été généré automatiquement le 23 avril 2019

Les ami•e•s de Variations 


\title{
Politisation de la parole : du rap ludique au rap engagé
}

\author{
Mathieu Marquet
}

\section{NOTE DE L'AUTEUR}

Merci à Julien Bordier pour la confiance et les relectures
«L'esclave est celui qui participe à la communauté
du langage sous la seule forme de la compréhension, non de la possession. »
Aristote, Politique, I

1 «A chaque débit de vers / On retrouve par terre des débris de verre / Jamais désolé si les mots font des victimes / et brisent le silence comme le pavé qui vise la vitrine » (Mokless 2011: "Cris d'guerre »), annonce en musique le groupe Scred Connexion. Miroir du mouvement social, le rap en a les images et le vocabulaire; les chants accompagnent les révoltes, on se révolte aussi en chanson.

2 En tant qu'expression populaire, ou expression $d u$ populaire, le rap porte un potentiel militant et contestataire. Mais s'agit-il d'une parole engagée qui est mise en musique ou bien d'une expression artistique qui se « politise » en chanson? A partir d'une quinzaine d'entretiens menés dans le cadre d'une thèse en cours de réalisation sur la question de l'engagement à travers le rap, nous traiterons de la question du devenir contestataire de la parole.

3 Nous montrerons d'abord en quoi le rap, à travers le discours qu'il véhicule, est porteur d'une dimension politique. Partant, la question que nous traiterons ne sera pas celle de la prise de parole, mais plutôt celle de son devenir résistant et militant : nous verrons qu'une partie des rappeurs qui tiennent un discours contestataire ont commencé le rap comme activité « ludique » et non pas subversive. On analysera alors comment cette parole a pu devenir politique. 
C'est donc un processus que nous nous étudierons ici : comment la prise de parole ellemême amène une conscience politique et une volonté de l'exprimer; comment, finalement, le fait d'avoir la possibilité d'exprimer ce que l'on pense amène à penser. ${ }^{1}$

\section{Le rap, une poésie rythmée et politique}

Le rap est né dans les années 1970 dans le contexte urbain des ghettos noirs américains, et s'inscrit plus largement dans le mouvement hip hop ${ }^{2}$. Il arrive en France au début des années 1980, et y connait un réel essor vers la fin de la décennie. Nous nous intéressons ici au rap français, que l'on peut définir à l'instar de son grand frère outre-Atlantique comme " une poésie rythmée (...), une épée à double face: elle tranche des deux côtés, tu peux couper des deux côtés, pas un seul, deux » [R. - entretien]. D’un côté le sens et la portée des mots, de l'autre leur rythme et leur musicalité.

6 Mais lorsque nous parlons de rap, à quel «type » de rap faisons nous allusion ? S'agit-il ici d'analyser l'ensemble des pratiques rassemblées sous cette appellation? Car, comme tout mouvement artistique et musical, le rap n'est pas homogène, et se compose de différents courants, tous interpénétrables : rap dit « conscient » (intérêt pour les questions sociales, dénonciation de l'ordre établi, revendication de l'égalité...), rap « egotrip» (qui renvoie principalement à de l'exercice de style), rap « festif » (dont les sujets récurrents seront les sorties nocturnes, les conquêtes amoureuses...) ou encore «gangsta » rap (qui privilégie les thèmes tels que la violence, l'usage d'armes à feu, la vente de drogue ...). C'est au rap dit «conscient» - que nous qualifierons indifféremment de "contestataire", " politique », ou encore " militant »- que nous nous intéresserons ici.

7 Toutefois, et avant de revenir sur le caractère politique de ce type de prise de parole, il convient de garder à l'esprit plusieurs éléments. En premier lieu, dans ce type de rap spécifique qu'est le rap contestataire, il est déjà possible de faire une distinction entre ceux qui pratiquent un rap «entièrement" politique, dans le sens où l'ensemble des thématiques qu'ils abordent ont pour but de dénoncer une situation ou de revendiquer certains droits, et ceux qui font un rap "varié ", avec, au sein de leur répertoire, des morceaux à caractère politique. Ce sont ces deux catégories de rap (et donc de rappeurs et de rappeuses) qui nous intéressent ici. En second lieu, précisons que si l'étude d'un tel objet relève bel et bien d'un choix, celui-ci est méthodologique : en aucun cas le rap ne peut se limiter à une activité de contestation, et on ne peut réduire cette musique à sa dimension subversive. Dès sa naissance, le rap est apparu autant comme forme festive (avec notamment son encrage dans les block partys, des fêtes de quartier) que comme forme d'engagement (en revendiquant par exemple sa filiation avec les Last poets, "poètes engagés» et "hérauts politiques" (Bonnet 2002: 75) du Harlem des années soixante-dix). Présenter le rap comme « expression de résistance » n'est pas une posture idéologique (faire de cette musique le porte-drapeau des révoltes populaires) mais sociologique : elle nous servira de tremplin pour étudier le processus de politisation de la parole.

Le caractère politique du rap est multiple. Il peut résider autant dans sa forme musicale, dans son vocabulaire, dans son mode de production que dans ses thématiques. Nous retiendrons pour notre part une de ces dimensions, à savoir le sens que les acteurs mettent dans leurs paroles. Le discours que véhicule le rap peut en effet être perçu et utilisé comme forme d'engagement par celles et ceux qui le pratiquent; il y a conscience 
et volonté d'agir sur les représentations et le monde social, que ce soit à travers une dénonciation de l'ordre politique établi, des conditions de vie d'une partie de la population française, ou encore la revendication d'une égalité universelle.

Pour développer cette idée, nous nous arrêterons sur une des thématiques militantes développées dans le rap, à savoir la volonté de proposer, à travers des textes diffusés sur un support musical, une autre Histoire, passée ou présente, une autre réalité sociale, historique ou contemporaine: apporter un contre-pied au discours dominant et médiatique. Cette volonté de réappropriation s'entend autant dans les textes mêmes des chansons, que dans les intentions de leurs auteurs, recueillies lors d'entretiens individuels. On peut citer, à titre d'exemple, la chanson «Mes héros» du rappeur Neg Lyrical, qui cite successivement des noms de « l'histoire Noire» (politiques, intellectuels, journalistes, militants ou activistes), explicitant leurs œuvres, leurs thèses et leurs combats. Toussaint Louverture, Aimé Césaire, Léopold Sédar Senghor, Gontran Damas, Frantz Fanon, le parti des Balck Panthers, Malcolm X, Martin Luther King, Rosa Parks, Marcus Garvey, Patrice Lumumba, Neslon Mandela, Cheikh Anta Diop, André Aliker ou encore Harriet Tubman traversent les couplets. Quant au refrain, il énonce :

Mes héros n'apparaissent pas sur les billets d'banques / Mes héros n'apparaissent pas sur le p'tit écran / Mes héros n'apparaissent pas dans leur enseignement / Où est-ce qu'ils les planquent? / Prenons en main l'éducation des nôtres / Sinon on attendra longtemps des autres (Neg Lyrical 2012 : « Mes héros »)

Dans la même veine, on peut également citer :

J'ai appris que même l'école fait des ravages / On te parle de Bonaparte comme une idole, mais pas de l'esclavage (Youssoupha 2009 : « Apprentissage »)

11 Ou encore cet extrait (tiré d'un album au titre équivoque, L'oubli pour mémoire), tentative de réappropriation d'une histoire sociale cette fois contemporaine :

Ils veulent cacher mon histoire au fond d'une armoire / Leur politique c'est l'oubli pour mémoire... Ils ont oublié / Que la France en 2013, on en est au même stade / Identité nationale, cris de singe dans les stades... Ils ont oublié / La pension des tirailleurs indigènes africains / Oubliée, la reconnaissance des travailleurs sanspapiers / Oublié, le droit de vote de nos citoyens immigrés (Youkoff 2013 : «L'oubli »)

Que ce soit par l'intermédiaire de supports physiques (les CD, par exemple), de «moments» spécifiques (tels que les concerts), par la diffusion (internet, radio, télévision...), ou encore par les réactions qu'il provoque (en attestent le nombre de prise de position d'hommes politiques ou des groupements idéologiques face aux paroles des rappeurs), ce type de rap a dans une certaine mesure pénétré l'espace public et il y circule. Or, « la sphère publique politique de l'Etat-social n'est qu'une forme dégénérée de l'espace public bourgeois » (Habermas 1962:241), réservée aux classes dominantes et à quelques « éclairés ». C'est ce que la pratique du rap remet en cause, en créant une forme originale d'espace public, un espace public «oppositionnel », que l'on peut définir à la suite de Negt comme l'ensemble des " formes alternatives et collectives donnant une expression publique aux besoins humains qui transgressent la cage d'acier des 
représentations dominantes, médiatisées.", espace qui concerne "tous les potentiels humains rebelles, à la recherche d'un mode d'expression propre » (Negt 2007 : 216, 222).

Le rap c'est un moyen de transmettre ce qu'on considère nous comme une vérité. La culture, il y a la culture dominante officielle, et la culture populaire. Nous, notre rap il est populaire, ça fait partie de ce qu'on nomme la culture populaire. Et donc c'est nous qui écrivons l'histoire là. C'est nous qui l'écrivons. C'est pas les dominants qui l'écrivent. Oui, moi j'estime que les mômes ne savent pas assez de choses sur l'histoire des luttes... et pourquoi ils savent pas que cet enfoiré de Jules Ferry c'était un esclavagiste par exemple? Ils ne savent pas plein de trucs. Donc nous notre rôle c'est de dire aussi « attention, il y a une autre histoire ». [S.1 entretien]

Ça a toujours été là, l'idée de parler d'une réalité qui n'existe pas médiatiquement, ou qui n'existe pas dans l'histoire telle qu'on nous la sert à l'école. Donc ça permet de régler nos lunettes, et de voir différemment l'histoire, ça c'est sur. Oui, ça peut permettre, l'art en général pas que le rap, de porter des améliorations à l'histoire, c'est-à-dire réajuster l'histoire, gommer les erreurs. Ça c'est sûr. [S.2 - entretien]

Du coup la musique peut devenir un acte, dans la mesure où elle renvoie à certaines bases, quoi. Ça peut être des actes, tout dépend l'audience et de ce que ça fait. Par les mots on peut faire beaucoup de choses. On change pas la situation, mais on y contribue dans la mesure où on injecte quelque chose, où on touche quelque chose chez un individu, qui l'amène après à agir. C'est un peu ça l'enjeu, c'est un peu ça le but, quoi [...]. On résout pas un problème en le décrivant, mais en tout cas, on amorce un processus de résolution de ce problème là. [A. entretien]

13 Deux éléments principaux transparaissent de ces extraits d'entretiens ${ }^{3}$ : le besoin de produire une Histoire différente, et l'importance de la nomination. Pour que cette autre Histoire puisse vivre, il faut nommer, exprimer des réalités et des noms jusque-là tus. Le fait de dire devient "acte ", prononcer fait exister. La dimension performative et politique est clairement recherchée à travers la démarche d'expression, l'enjeu étant de toucher les individus autant que d'agir sur des représentations sociales. Dans une certaine mesure, ce type de rap représente alors une "généralisation" de l'espace oppositionnel décrit plus haut. En effet: "De nos jours, la pratique oratoire ne trouve malheureusement un écho significatif que lorsque se développent de véritables mouvements de révolte, comme cela a été le cas lors du mouvement pacifiste allemand ou lors des vagues de manifestation de 2006 en France. Ce qui manque est la généralisation quotidienne de cette pratique de l'espace public oppositionnel. » (Negt $2007: 219$ )

Dans sa conception, sa pratique et sa diffusion, le rap actualise un espace public de résistance et le rend pérenne. Le discours de certains rappeurs donne une nouvelle dimension à la " pratique oratoire ", dans le sens où la parole contestataire se diffuse en permanence, et non plus seulement lors de mouvements sociaux ponctuels.

15 La pratique du rap n'est pas uniquement une pratique artistique, mais bien une pratique sociale, un fait social et politique. Selon cette définition du rap (qui, rappelons-le, ne correspond qu'à une des ces composantes de ce mouvement délimitée par notre analyse), l'individu qui s'inscrit dans ces processus de mise en mots rythmés devient un acteur militant, dans le sens où " l'interaction militante, en même temps qu'elle est une contribution matérielle au mouvement et à la solidarité, est échange dans les mots, communication et possibilité de parler dans d'autres termes ce qu'on est et ce qu'on vit. » (Cingolani 2003 : 128)

Je me suis rendu compte qu'un texte me permettait de dire ce que d'autres n'auraient pas dit de la même manière, et que ça avait un impact. Que voilà, en entendant un texte, les gens, mine de rien, ils prennent conscience de quelque chose, ou pas, mais ils entendent. Et tu fais quelque chose de concret, même avec des mots, avec une voix. C'est pour ça que le rap, c'est 
la place privilégiée; naturellement le rap c'est placé comme le genre d'une révolte, d'une insoumission. [H. - entretien] cherchent, à travers leur discours, à produire des effets, à remettre en cause certains schèmes de pensée, et participent ainsi à l'élaboration d'un espace oppositionnel. Le travail sur la construction de l'Histoire nous en donne une illustration concrète. Mais ces mots sont-ils « naturellement » revendicatifs, comme il est suggéré dans l'entretien cité cidessus? Le discours des rappeurs est-il, dès leur prise de parole, politique par essence ? Comment la prise de parole, en elle-même et par elle-même, peut mener à une politisation et une volonté de l'exprimer?

\section{La parole ludique des débuts}

17 Il apparaît au travers des entretiens qu'une partie des rappeurs et rappeuses interrogé-e$\mathrm{s}$ - qui pratiquent un rap politique ou comptent parmi leur répertoire rapologique des textes politiques - n'a pas commencé sa «carrière » sur une prise de parole militante. La quinzaine d'entretiens réalisée ne nous permet pas pour l'instant d'élaborer une typologie définitive des modes de politisation de la parole. Néanmoins, un schéma se dessine : les rappeurs et rappeuses qui appartiennent à une famille déjà politisée et qui ont connu durant leur éducation des formes de socialisation politique, formelles ou informelles (participation à des manifestations, parents engagés dans des actions politiques, syndicales et/ou militantes, présence de livres à caractère politique au sein du foyer, souvenirs d'enfance de débats à la maison...), sont rentrés dans le rap notamment parce qu'ils avaient "quelque chose à dire », la volonté d'exprimer un «message». A l'inverse, il semble que pour les individus plus éloignés de ces formes traditionnelles ou nouvelles de culture politique, le rap constitue à son origine une pratique ludique, récréative, voir «sportive ». Ce n'est que dans un deuxième temps que le discours prend entièrement ou en partie - un caractère contestataire.

- D. : Moi les premiers textes que j'ai fait c'était en anglais carrément. C'était juste pour l'exercice, du flow, et tout. C'était pas du tout pour être exposé de toute façon, mais je m'étais amusé dans ma tête : j'étais livreur à Pizza Hut, j'étais sur ma mobylette toute la journée, du coup je m'étais fait un texte, avec que des phrases toutes faites, mais en anglais! Et après, les premiers textes que j'ai fait en français, je cherchais vraiment à retrouver, parce que j'aimais le rap, ce qu'untel avait réussi à faire sur son dernier flow. Mais je pensais moins à qu'est ce que je vais dire ou est-ce que ma phrase elle est... Le premier truc c'était vraiment forme, forme, forme, et après bah tant mieux si tu dis des bons trucs. Deuxième texte tu commences à... C'est petit à petit que c'est arrivé. J'suis pas un mec qui écrit des bouquins et puis après du rap. Les premières fois que j'écrivais, c'était plus pour la forme, et après je me suis rendu compte que c'était un biais qui me permettait aussi d'aller plus loin.

- Donc toi t'es plus venu au rap pour la forme?

- D : Ouais, pour s'amuser, au début c'était un peu un divertissement. On aurait pu se mettre au rugby, ou au skate... Tu vois le rap c'est une activité cool entre potes. T'as besoin de rien...

- B : C'est sportifle truc!

- A la base c'était plus comme un sport plutôt qu'un besoin d'exprimer quelque chose?

- D : Ouais, je savais pas que j'avais des choses à dire. Je m'amusais, et puis au fur et à mesure, tu dis des trucs quoi.

- B. : Tu te prends au jeu, on se prend au jeu. C'est un truc qu'est pas prévu, ça arrive, tu commences ça comme un sport, tu te dis « ah putain l'autre il a bien rappé, il a fait un bon 
flow, il fait un bon thème... ». Et au final tu te rends compte que bah ouais, c'est cool quand même, d'avoir cet exutoire, de pouvoir exprimer ce que tu vis, que ce soit la paternité, les galères de couple, ou la vie, les hauts et les bas. Le fait d'avoir cette soupape, de pouvoir exprimer quelque chose par rapport à la réalité, le quotidien, le côté pesant du quotidien. Et le rap c'est vachement à propos de ça. [D. et B. - entretien]

Moi personnellement je suis pas arrivé au rap via le côté engagé, j'avoue. J'ai écouté des choses qui m'ont plu, c'est la rythmique. C'est ça qui m'a plu à la base. Mais au fur et à mesure, à force d'écouter des choses, je me suis rendu compte que... J'ai beaucoup appris par le rap. Donc mon discours a muté à un moment vers le côté plus politique, mais à la base, c'était pas le côté politique. [E. - entretien]

Si le rap était par essence l'expression d'une révolte, comment expliquer qu'une partie de ceux qui scandent des paroles revendicatives l'ont pratiqué à leurs débuts dans une optique "récréative»? La dimension politique et contestataire de la parole n'apparaît que dans un deuxième temps. C'est le fait même de pouvoir dire qui mène vers une envie de dire, et partant, à l'expression du et d'un point de vue politique.

\section{Les facteurs explicatifs de la politisation de la parole}

S'intéresser à cette expression artistique peut alors «nous aider à comprendre les processus par lesquels les nouvelles forces et les nouvelles revendications politiques germent avant de venir fleurir la scène » (Scott 2009 : 216), comprendre comment la parole devient politique, comment se forment les espaces de parole contestataires.

Pour Karim Hammou, auteur d'une récente Histoire du rap en France, on peut expliquer le développement des prises de positions contestataires des rappeurs français par un mécanisme de co-construction. Acteurs du mouvement, médias et politiques ont conjointement contribué à l'assignation du rap aux thèmes de la "banlieue », de la "rue ", à des "problématiques sociales " (et par là notamment le discours affirmatif d'une dignité, qui passe entre autre par la redéfinition de l'Histoire). En effet, le rap prend une dimension médiatique nationale à partir de 1990, et ce principalement à travers la télévision. Il est alors associé au «tag »- qui devient dans ces années un problème public - ainsi qu'à la question des "bandes", qui préoccupent alors le discours dominant. La médiatisation de pratiques et phénomènes sociaux émergeants jugés vandales et incivils braque les projecteurs sur le mouvement hip hop français naissant. Les rappeurs sont alors utilisés par la télévision comme représentants, porte d'accès et porte-paroles de ces nouvelles classes dangereuses. Parallèlement, ces pratiques urbaines (graffiti, danse, rap... ) sont récupérées par les politiques de la ville, qui voient là un moyen de juguler les problèmes de certains quartiers dits difficiles et de canaliser une jeunesse marginalisée ${ }^{4}$. On peut donc dire, avec Hammou, qu'à partir du début des années 1990 : «L'assignation médiatique du rap aux banlieues et l'ancrage du hip-hop dans les quartiers de la politique de la ville interagissent ainsi avec l'expérience sociale d'une frange de la jeunesse, dans un contexte de paupérisation des quartiers populaires, de ségrégation spatiale accrue et de tournant répressif dans la gestion des illégalismes populaires. Ils contribuent à légitimer l'élaboration musicale de formes d'écriture, de points de vue et de thèmes nouveaux. » (Hammou 2012 : 141)

Lorsque l'on cherche à expliquer comment une frange du mouvement s'est chargée d'un contenu contestataire, il faut donc prendre en compte l'influence des médias ainsi que de certaines mesures politiques. On ne peut toutefois expliquer ce phénomène de manière unilatérale, et la marge de manœuvre des individus face aux tentatives de définition 
normatives reste essentielle. Il s'agit alors de voir comment les acteurs eux-mêmes ont, en fonction de ces assignations ou indépendamment, développé la dimension subversive de leurs textes ${ }^{5}$.

Une des raisons de cette prise de parole politique n'est autre que la volonté, première et indépendante des définitions dominantes du rap, de dénoncer certaines injustices en musique et de militer à travers la culture. C'est notamment le point de départ des individus politisés avant même de commencer le rap. Ici, la prise de parole contestataire, en tant que mobilisation individuelle, suit le parcours "classique »du militantisme: sentiment d'injustice et envie de revendication mènent à prendre la parole. Mais qu'en est-il de ces rappeurs qui sont rentrés dans le rap sans un ethos militant préalable? Quatre autres facteurs de politisation de la parole apparaissent au travers de nos entretiens ${ }^{6}$.

Le devenir militant du discours dans le rap s'explique premièrement par les trajectoires et les expériences individuelles, l'apprentissage de la vie nourrissant l'inspiration artistique.

- Donc t'as pas commencé le rap par un « message »?

- Bah nan, parce que quand t'as quinze ans, au tout début... C'est plus dans ma deuxième partie de rap où vraiment, avec l'âge, t'as des prises de consciences, tu deviens majeur, y'a des trucs qui t'arrives dans ta vie, tu rencontres le travail, les difficultés de la vie, et tout ça, ça t'amène aussi à de la connaissance, parce que les problèmes que tu rencontres dans ta vie, tu vas forcément en parler, donc à un moment tu les mets en musique. Et je pense que l'écriture ça s'apprend toujours, parce que dans ta vie, tu as toujours des choses qui te tombent dessus, dont tu parleras, donc forcément tu apprendras toujours. Si tu apprends dans la vie, tu apprendras dans l'écriture. [C. - entretien]

Faire l'épreuve de certaines réalités permet donc au discours de se charger d'une nouvelle dimension sociale ; prosaïquement, cela amène les acteurs à exprimer des choses dont ils n'avaient pas forcément conscience avant de les vivre.

Ensuite, la recherche d'informations complémentaires pour les besoins de l'écriture permet d'acquérir de nouvelles connaissances, de s'ouvrir à de nouvelles thématiques, et ainsi de développer et affirmer son engagement.

L'écriture m'a énormément apporté, je me suis cultivé en cultivant mes textes. Je suis rentré dans un cercle un peu "vertueux", où j'écris des textes sur des sujets qui me passionnent et je lis des livres pour vérifier que j'ai pas dit de conneries; du coup le sujet me passionne encore plus et j'ai encore plus de choses à dire pour faire d'autres morceaux. C'est pas mal pour briller en société aussi, par exemple: j'écris un truc sur le nucléaire (donc je matte des films, je lis des sites, des livres etcétéra...), ensuite j'apprends par cour mon lyrics et je suis capable de ressortir les points importants en trois coups de cuillère à pot parce que j'ai bien bossé le sujet. Je nourris mon discours et ça me met souvent en position de pouvoir à mon tour nourrir le débat. Oui, on peut travailler notre propre pensée, ça parait très dur mais c'est possible et on y prend goût. [D. - entretien]

Troisièmement, c'est le média utilisé lui-même qui peut mener à la revendication. En d'autres termes, le genre musical sur lequel s'appuie tel ou tel type de discours influence le contenu de ce dernier. Concernant le rap, c'est notamment sa forme chansonnière qui ouvre une fenêtre au militantisme, dans le sens où la chanson de rap est un espace musical et rythmique où la part laissée aux mots est importante. Le format invite en quelque sorte le «sens » à s'adjoindre au jeu, le fond à la forme.

- D. : La particularité aussi, c'est qu'en général, même si y'a des rappeurs qui ont des flows

très lents, y'a beaucoup de place pour les mots dans cette musique là, plus que dans du chant 
où les syllabes vont être plus étirées, donc tu pourras dire un peu moins de mots dans ta chanson. Donc à partir du moment où t'as beaucoup de place pour les mots...

- B. : Faut du sens!

- D. : La particularité par rapport à d'autres disciplines c'est que t'as beaucoup de place, par rapport à un écrivain, non... mais par rapport à de la musique, donc tu peux... C'est plus qu'un format chanson, ça se rapprocherait presque d'un article en termes de quantité de mots, ou d'un p'tit bouquin quand il s'agit d'un album. [D. et B. - entretien] enfin, cet espace de liberté et d'égalité que le rap offre à ses adeptes qui permet de comprendre comment des mots se dotent d'une charge politique. En plus de créer un espace public oppositionnel - et même plus : pour créer cet espace public oppositionnel -, le rap créé un espace social qui permet de libérer une parole potentiellement soumise aux enjeux de la hiérarchie sociale. En effet, « l'idée que les êtres parlants sont égaux par leur capacité commune de parler est une idée raisonnable/déraisonnable, déraisonnable par rapport à la manière dont se structurent les sociétés, depuis les antiques royautés sacrées jusqu'aux modernes sociétés d'experts. » (Rancière 1995 : 85) Malgré les principes affichés d'égalité des droits et de liberté d'expression, la parole et son usage sont aujourd'hui inégalement répartis en fonction des appartenances sociales. Ainsi, comme le souligne Bourdieu, dans nombre de cas « l'autorité advient au langage du dehors » (Bourdieu 1982 : 105) ; cela revient à dire qu'un discours ne produit d'effets qu'à partir du moment où son producteur a la légitimité de l'exprimer. Cette situation de domination à travers le langage est telle qu'elle empêche une partie de la population de formuler opinions et vision du monde, voire même de les concevoir.

En situation de domination, la production d'idées - surtout lorsqu'elles sont subversives peut alors prendre la forme de ce que James $C$. Scott a appelé le « texte caché ». «Les sites du texte caché, nous dit-il, sont les lieux dans lesquels la riposte tue, la colère retenue et les langues restées liées à cause des relations de domination trouvent un vecteur d'expression véhémente et à gorge déployée. ( (Scott 2009 : 135) Or, ce texte se développe «lorsqu'il est articulé dans un espace social protégé imperméable au contrôle, à la surveillance et à la répression du dominant. » (Ibid.) Le texte caché permet donc une critique du pouvoir, une remise en cause l'ordre établi qui se fait d'abord « en coulisses », avant, dans certains cas, de passer sur la scène publique.

Le rap, ça t'auto-éduque. Ça t'auto-éduque, et ça éduque les autres, c'est ça qu'est fort avec les mots. Et personne peut te couper. Quand tu mets lecture sur un son, y'a personne pour te couper la parole et te dire " ouais, mais... ». Nan, t'es obligé d'écouter le son jusqu'à la fin. Et c'est ça qu'est fort dans un son. Et surtout dans le rap. Quand t'as un vrai message à passer et que tu fais du rap, c'est ça qu'est fort : la personne peut pas te couper. Alors que dans une discussion, y'aura toujours quelqu'un qui aura plus d'aisance que toi et qui te fera passer pour un débile, même s'il a moins de vocabulaire que toi. Mais dans un morceau, personne peut te couper la parole, c'est ça qu'est bien. Et la plupart de ces sociologues à la con, eux comme ils ont l'habitude des débats, ils vont réussir à te faire fermer ta gueule facilement parce que... l'intimidation du discours. L'oration et l'intimidation dans le discours va faire que toi tu vas fermer ta gueule. Mais ça veut pas dire que t'es plus te-bê [bête] que lui, parce que dans un autre contexte tu serais capable peut-être de t'exprimer beaucoup mieux. Et même de lui faire comprendre des choses. [...] Quand tu écris, y'a pas un autre mec avec un stylo qui intervient sur ta feuille qui dit "nan, dégages ton stylo, j'vais écrire à ta place». Nan, c'est toi qui es devant ta feuille. [C. - entretien]

En tant qu'espace de création, le rap permet la formulation du texte caché et sa libération. Il créé un contexte qui permet la mise en mot politique des conditions d'existence, un espace qui neutralise, le temps de l'écriture au moins, les mécanismes de 
domination de la parole et les effets de la hiérarchie sociale ${ }^{7}$. "Puisque la résistance idéologique a les meilleures chances de se développer lorsqu'elle n'est pas soumise à une surveillance directe, on est amené à examiner les espaces sociaux les plus propices à cette résistance » souligne Scott (1990: 13). Et le rap est un de ces « espaces sociaux»; espace de résistance privé qui forme un espace public oppositionnel, passant du texte caché au texte manifeste, et de l'individuel au collectif.

Cette écriture, qui ouvre de nouveaux espaces et par là une nouvelle vision du monde, donne à voir le caractère performatif des mots, cette « puissance d'agir » du langage qui a pour effet de permettre à l'individu de sortir de sa condition et de porter un discours qu'il n'était pas censé produire. Cette perspective nous invite alors à penser «la force insurrectionnelle de l'émergence du discours censuré dans le "discours officiel", qui ouvre le performatif à un futur imprévisible » (Butler 2004 : 222), futur qui redéfinit en partie le partage de la parole.

\section{Espaces libérés pour une parole émancipée}

31 Un certain type de rap peut être qualifié de politique de par le discours et les intentions de celles et ceux qui le pratiquent. L'expression en musique d'une autre réalité (et notamment la redéfinition de l'Histoire, passée ou contemporaine) créent un « espace public oppositionnel » et le fait vivre. On peut à cet égard parler de discours subversif et de démarche militante. Mais, pour une partie des interviewés (parmi lesquels on retrouve principalement des individus sans forte socialisation politique initiale), la dimension contestataire n'est apparue que dans un deuxième temps, après une approche plus «ludique». Plusieurs facteurs permettent d'expliquer cette politisation de la parole émise. L'influence des médias et des politiques publiques, les expériences de vie individuelles, les recherches thématiques que nécessite l'écriture, la forme chansonnière spécifique du rap, et enfin l'espace de liberté et d'égalité qu'il procure sont autant d'éléments qui expliquent le devenir politique de cette parole ${ }^{8}$.

Le rap, en tant que prise de parole, est en soi un facteur explicatif de l'engagement individuel. Il existe donc un pouvoir performatif du langage: par la force de la nomination, ce dernier tend à former de nouvelles catégories de pensée, de nouveaux cadres de représentation, collectifs comme individuels; car les mots ont également le pouvoir d'amener l'individu qui s'en empare à un changement de perspectives (par le développement d'une conscience politique notamment), voire de situation (passer de l'engagement militant en chanson à d'autres formes d'engagements, par exemple). Ce changement, nous l'avons dit, passe notamment par l'espace social que l'écriture peut créer, espace privilégié, "protégé », qui permet à l'individu de se libérer des mécanismes de domination inhérents à l'usage des mots. Cette brève analyse du discours politique à travers le rap montre alors comment peut se former, à partir de la prise de parole, une posture contestataire. C'est lorsque l'on acquiert, dans un contexte particulier, la possibilité de réfléchir à sa situation et à celle de son environnement social que la réflexion et le discours peuvent se faire politiques. Pour pouvoir dire ce que l'on pense, il faut avoir la possibilité de penser ce que l'on va dire. Cela confirme l'importance fondamentale de l'aménagement d'espaces sociaux et culturels à même de libérer la parole de chacun, des espaces qui (re)donnent aux mots tout leur pouvoir. 


\section{BIBLIOGRAPHIE}

Bonnet, Valérie. 2002. «Revendication et politiques en paroles : chansons de la communauté noire américaine », Mots. Les langages du politique [En ligne], 70, 65-78

Bourdieu, Pierre. 1982. Ce que parler veut dire. L'économie des échanges linguistiques (Paris : Fayard)

Butler, Judith. 2004 [1997]. Le pouvoir des mots. Politique du performatif (Paris : Editions Amsterdam)

Cingolani, Patrick. 2003. La république, les sociologues et la question politique (Paris: Editions La Dispute)

Habermas, Jürgen. 1962. L'espace public. Archéologie de la publicité comme dimension constitutive de la société bourgeoise (Paris : Payot)

Joana, Jean. 1994. « Les usages de la méthode biographique en sciences sociales », Pôle Sud, vol. 1, $\mathrm{n}^{\circ} 1,89-99$

Mokless. 2011. Le poids des mots (Y\&W Prod / Because Music)

Neg lyrical. 2012. Explicit Lyrics (Neg lyrical)

Negt, Oskar. 2007. L'espace public oppositionnel (Paris : Payot)

Rancière, Jacques. 1995. La Mésentente. Politique et philosophie (Paris : Galilée)

Scott, James C. 2009 [1990]. La domination et les arts de résistance. Fragments du discours subalterne (Paris : Editions Amsterdam)

Youkoff. 2013. L'oubli pour mémoire (La Baraka Prod)

Youssoupha. 2009. Sur les chemins du retour (Bomayé Musik / EMI Music France)

\section{NOTES}

1. En terme de méthodologie, nous avons essayé de laisser dans cet article une place certaine aux extraits d'entretien, que nous n'avons par ailleurs que rarement analysés en tant que tels. Cela se justifie dans la mesure où l'usage des entretiens n'est pas uniquement illustratif. Les propos recueillis ont ici valeur de raisonnement sociologique : ils ne sont plus uniquement le matériau à expliciter, ils sont aussi partie prenante du raisonnement.

2. Il en est l'une des quatre disciplines artistiques, avec le deejaying (mixage de musique sur platines), le break dance (danse) et le graffiti (« aerosol art »).

3. Un troisième point central mériterait tout de même d'être souligné : la conscience des acteurs de n'être que producteur d'un discours, et non pas acteur de terrain ; l'idée que l'acte de parole « amorce » une action, qu'elle soit la leur ou celle d'autres militants.

4. On peut rajouter une dernière "étape » à ce processus : le développement par les majors de l'industrie du disque et certaines radios nationales, vers 1998, d'un "marketing de la marge » (Hammou 2012 : 182), encourageant sur fond d'étude de marché un rap plus « rue », plus dur et plus revendicatif.

5. C'est typiquement le genre de mécanisme que peut nous permettre de comprendre l'enquête par entretien de type biographique, qui « va s'appliquer à mettre au jour le jeu entre individu et 
contraintes objectives. Elle va être le moyen de mesurer le poids des normes sur les individus d'un même groupe, les rapports de forces entre normes concurrentes, les possibilités de choix laissées aux sujets et la contribution qu'ils apportent à l'évolution de ces normes » (Joana 1994 : 94).

6. On pourrait en citer un cinquième, en poursuivant avec la thèse de Karim Hammou : certains rappeurs vont, en réponse à l'utilisation qui est faite de leur image dans les médias et par les acteurs du monde politique, assumer ce mandat de "représentant», et faire des questions sociales - urbaines - leur thème de prédilection.

7. Ce contexte spécifique de libération de la parole permet également de neutraliser d'autres types de domination et de barrières que peut rencontrer l'expression. Ainsi, certains interviewés expliquent avoir eu recours à l'écriture et au rap pour déclamer ce qui leur était impossible de dire en d'autres situations: exprimer des sentiments amoureux ou un ressenti quant aux relations familiales par exemple.

8. Il est intéressant de noter que les quatre facteurs que nous avons mis en lumière influent aussi bien sur ceux qui ont commencé le rap par une pratique festive que sur ceux qui ont commencé avec dès le début la volonté d'exprimer un message contestataire. Cette généralisation est possible car les mécanismes sont analogues: dans le premier cas, ils amènent à la formation d'une conscience politique et la production du discours revendicatif ; dans le deuxième cas, ils conduisent à l'affirmation et au développement d'un l'engagement préexistant.

\section{AUTEUR}

\section{MATHIEU MARQUET}

Doctorant en sociologie, Université Paris X Nanterre 